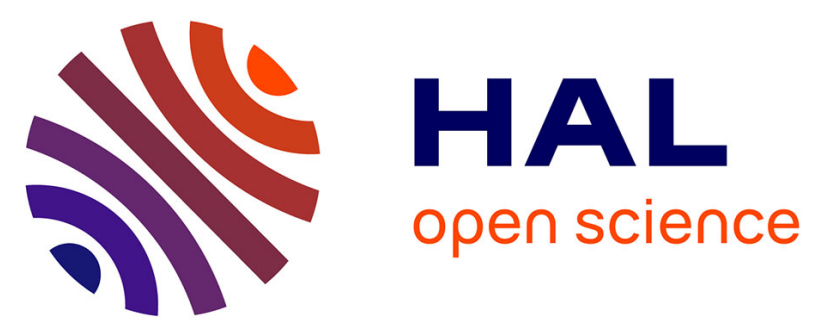

\title{
Le recours aux dossiers des patients est-il nécessaire pour l'évaluation du risque de récidive sexuelle, violente et générale?
}

Ingrid Bertsch, Robert Courtois, Christian Réveillère, Thierry Hoang Pham

\section{- To cite this version:}

Ingrid Bertsch, Robert Courtois, Christian Réveillère, Thierry Hoang Pham. Le recours aux dossiers des patients est-il nécessaire pour l'évaluation du risque de récidive sexuelle, violente et générale?. L'Encéphale, A paraître. hal-03280121

HAL Id: hal-03280121

https://hal-univ-tours.archives-ouvertes.fr/hal-03280121

Submitted on 17 Sep 2021

HAL is a multi-disciplinary open access archive for the deposit and dissemination of scientific research documents, whether they are published or not. The documents may come from teaching and research institutions in France or abroad, or from public or private research centers.
L'archive ouverte pluridisciplinaire HAL, est destinée au dépôt et à la diffusion de documents scientifiques de niveau recherche, publiés ou non, émanant des établissements d'enseignement et de recherche français ou étrangers, des laboratoires publics ou privés. 
Le recours aux dossiers des patients est-il nécessaire pour l'évaluation du risque de récidive sexuelle, violente et générale?

Is the use of patient records necessary to assess the risk of sexual, violent and general recidivism?

\section{Ingrid Bertsch ${ }^{1,2,4 *}$, Robert Courtois ${ }^{1,2}$, Christian Réveillère ${ }^{1}$, Thierry Pham ${ }^{3,4}$}

\footnotetext{
${ }^{1}$ Université de Tours, département de psychologie, EE 1901 Qualipsy (Qualité de vie et santé psychologique), 37000 Tours, France.

${ }^{2}$ Centre Ressource pour les Intervenants auprès des Auteurs de Violences Sexuelles Centre Val-de-Loire, CHRU de Tours, 37000 Tours, France

${ }^{3}$ Centre de Recherche en Défense Sociale, CRDS, 7500 Tournai, Belgique.

${ }^{4}$ Université de Mons-Hainaut, département de psychologie légale, 7000 Mons, Belgique
}

* Auteur correspondant : Ingrid Bertsch

CRIAVS, hôpital Trousseau, CHRU de Tours, avenue de la République, 37044 Tours cedex 9

Email : $\underline{\text { i.bertsch@chu-tours.fr }}$ 


\section{Résumé}

Introduction : La diversité des sources d'informations recueillies lors des évaluations du risque de récidive 1 sexuelle, violente ou générale d'infracteurs sexuels et/ou violents est primordiale. En France, un accès limité 2 aux informations contenues dans les dossiers médicaux et pénaux pourrait entraîner une utilisation erronée de 3 ces échelles. L'objectif de l'étude est de tester l'utilité du recours au dossier médical pour la cotation d'échelles 4 de risque de récidive sexuelle, violente et générale. Matériel et Méthode : Cent vingt-huit auteurs de 6 violences ont été évalués à l'aide de trois échelles de risque de récidive sexuelle, violente et générale. Les 7 scores et les items des échelles ont été comparés selon $(a)$ qu'ils aient été cotés avec les dossiers médicaux et 8 un entretien ou $(b)$ seulement avec un entretien. Résultats et discussion : Des différences ont été observées ement chez les agresseurs sexuels et pour les échelles RSVP et LS/CMI. L'utilisation de dossiers médicaux atténue le poids des facteurs présents et futurs, majore le poids des facteurs passés et de facteurs qui peuvent entraîner plus d'émotions négatives chez l'évaluateur. Le fonctionnement psychique des personnes évaluées, l'attitude de l'infracteur et la nature de certaines informations sont discutés afin d'éclairer ces résultats. Conclusion : L'accès aux informations des dossiers des personnes évaluées devrait être questionné en France pour une utilisation éthique des échelles d'évaluation de risque de récidive.

Mots-clefs : Dossier de soins ; Évaluation ; Risque de récidive ; RSVP ; LS/CMI.

\section{Summary:}

Introduction: Sexual, violent and general recidivism risk scales are widely used in a number of countries. Their psychometric qualities are generally considered to be good. However, in practice they may vary in the quality of prediction of risk of sexual, violent and general recidivism, in particular because of the sources of the information collected. In France, the medical records of incarcerated patients are kept by health-care professionals. Although regulated, the content and quality of these records vary widely from one patient to another. The criminal justice system holds the criminal records of convicted and imprisoned persons. There is no set list of documents contained in these records. For caregivers and researchers, access to criminal records is difficult because of the confidentiality to which legal professionals are subject. The aim of our study was to investigate whether using medical files in addition to structured interviews can improve the assessment and management of the risks of sexual, violent and general recidivism. Material and Method: 128 perpetrators of violence were assessed using three scales of risk of sexual, violent and general recidivism. Scores for the items of the scales were compared between (a) those that were based on medical records and an interview, and (b) those based only on an interview. Results and discussion: First, differences in scores between the two groups (assessed through interview only, and assessed through interview and use of medical records) were observed on the RSVP, HCR-20 and LS/CMI scales. Secondly, most of the results indicate that the overall level of risk was perceived as lower when medical records were used, which would, indirectly, lead to a reduction in false positives when evaluating perpetrators of sexual violence. Thirdly, the point-by-point analysis shows that the use of information contained in the medical records reduces the weight of present and future factors (e.g. the physical and psychological stress of recent events), increases the weight of past factors (e.g. history of sexual violence), and can increase the weight of certain factors that can lead to more negative emotions in the assessor (e.g. deviance). These results can be explained by (i) the emotional functioning of the persons assessed (particularly defensive processes or memory difficulties), (ii) the attitude of the aggressor (particularly the presence of emotional and cognitive biases), (iii) the nature of the information (particularly "hot" cognitions or those leading to greater social desirability). The limitations of the study concern the relatively small number of participants, the environment in which the file was transmitted and the very heterogeneous and sometimes relatively incomplete composition of the files. Conclusion: The use of information contained in medical files impacts the results of recidivism risk scales and restores a balance to the factors. In France, risk scales are currently being introduced, although their use is still limited in the health field. However, ethical use of these scales raises the issue of the homogenisation of the content of medical records and access to criminal records in order to enable future research to confirm whether the use of information provided in medical and criminal records can improve the quality of assessment and treatment of offenders.

Keywords: Medical record; Assessment; Recidivism risk; RSVP; LS/CMI 


\section{INTRODUCTION}

L'évaluation du risque de récidive sexuel, violent et général chez les infracteurs violents est une

1 2 3 4 5

préoccupation majeure depuis plusieurs décennies. Le jugement clinique non structuré a été, et est encore dans certains pays comme la France, la méthode la plus couramment utilisée. Elle consiste à donner son avis sur le risque de récidive d'un patient uniquement à partir d'informations internes c'est-à-dire de l'expérience personnelle de l'évaluateur. Cette méthode d'évaluation s'est avérée peu efficace car l'évaluation du risque fait intervenir de nombreux biais. L'état du patient évalué, le contexte et les enjeux de l'évaluation, le lieu et le temps consacré à celle-ci ou encore la crainte des conséquences d'une mauvaise évaluation sur une institution impactent la qualité de la prédiction [1]. À ces difficultés, il semble primordial d'ajouter toutes les caractéristiques de l'évaluateur qui influencent l'évaluation [2] et notamment ses biais cognitifs. Par exemple, le biais de disponibilité conduirait l'évaluateur à penser que ce qui est saillant est plus fréquent et donc plus lié à la récidive [3]. L'ensemble de ces biais amènent l'évaluateur à prédire un risque de récidive plus élevé qu'il ne l'est en réalité (par exemple, [4]).

Pour contrer ces difficultés et permettre une évaluation plus juste du risque de récidive, des échelles structurées ont été développées. Elles s'appuient sur des informations externes que sont les facteurs empiriquement liés au risque. Ces facteurs de risque sont multiples et peuvent être judiciaires (comme, les antécédents d'infractions ; par exemple [5]) ou cliniques (comme, la présence d'un trouble de la personnalité ; par exemple, [6]). Les échelles de risque de récidive de jugement professionnel structuré (dites de dernière génération) sont devenues pratiques courantes dans le monde et notamment en Amérique du nord et en Belgique [7-9]. Elles ont pour objectif $(i)$ de prédire le risque d'une personne de commettre une infraction sexuelle et/ou violente (par exemple, [10]) et ou (ii) de mettre en place une prise en charge sanitaire et judiciaire adaptée pour les infracteurs (par exemple, [11]). Elles présentent souvent de bonnes qualités psychométriques qui les rendent supérieures au jugement clinique non structuré [12]. Toutefois, leur simple usage ne garantit pas une évaluation précise du risque.

Ainsi, le risque est évalué avec plus de précisions lorsque des professionnels sont formés aux échelles $[13,14]$, qu'ils se montrent plus consciencieux (en menant des évaluations plus détaillées et précises) [15] et qu'ils procèdent à l'évaluation dans un contexte favorable (notamment lorsqu'un lien de confiance est possible entre les deux protagonistes de l'évaluation) [16]. L'évaluation présentera plus de rigueur lorsqu'elle est 
réalisée de manière consensuelle avec plusieurs évaluateurs [17]. La validité de l'évaluation est également conditionnée par la qualité des informations recueillies. D'une manière générale, l'information recueillie

1

influence la prise de décision [18]. En ayant accès à des sources d'informations multiples, l'évaluateur aura davantage de facilité à coter les items pour lesquels les informations convergent [19]. Des informations identiques provenant de sources multiples vont avoir pour but de donner à l'évaluateur plus de confiance dans sa cotation qui a son tour entraînera une prédiction plus précise du risque de récidive [20]. Selon Guay [16], une bonne qualité d'informations recueillies comprend la qualité de l'entretien clinique mené auprès de l'évalué mais aussi l'accès à son dossier pénal.

En France, les personnes condamnées et incarcérées sont encadrées par l'Administration Pénitentiaire (chargée du bon déroulement de la détention), les magistrats (chargés de faire appliquer la loi) et le Service Pénitentiaire d'Insertion et de Probation (SPIP) (chargé de faire exécuter les mesures de justice). L'ensemble des professionnels judiciaires sont employés par le Ministère de la Justice. Les dossiers pénaux constitués par ces professionnels regroupent l'intégralité des pièces de procédure liées à une affaire. Bien qu'aucune liste fixe n'existe concernant les documents contenus dans les dossiers pénaux, les informations doivent permettre (i) la représentation des événements de l'affaire et (ii) l'attestation de ces événements [21]. Il peut donc contenir : des procès-verbaux d'interpellation, de condamnation, de constatation, d'audition, les expertises psychologique et psychiatrique... Pour les soignants et les chercheurs, l'accès aux dossiers pénaux est rendu difficile par le secret auxquels sont soumis les professionnels judiciaires [22].

Mais, ce sont surtout les dossiers médicaux qui peuvent être accessibles aux chercheurs français provenant de milieux de soin. Sur le plan médical, les personnes condamnées et incarcérées sont prises en charge par des professionnels du Ministère de la Santé. La constitution et la tenue d'un dossier médical sont obligatoires pour le médecin et les professionnels paramédicaux qui sont, avec le patient, les seuls à pouvoir accéder à ces informations [23]. Ce dossier peut contenir $(i)$ les documents établis au moment de l'admission et durant le séjour médical (par exemple, les prescriptions initiales, le type de prise en charge reçue, la nature des soins dispensés, l'état clinique...), (ii) les documents établis à la fin de chaque séjour hospitalier (par exemple, le compte rendu d'hospitalisation, les prescriptions établies à la sortie du patient, la fiche de synthèse des soins infirmiers...) et (iii) les informations recueillies auprès d'un tiers [24,25]. Cependant dans la pratique quotidienne, le contenu des dossiers médicaux est très hétérogène selon les patients et les établissements. 
Cet article a pour objectif de questionner les différences de cotation et d'interprétation des échelles de risque de récidive (a) à l'aide d'un entretien semi-structuré ou (b) avec un entretien semi-structuré et

1 2 3

l'exploitation des dossiers médicaux. Nous formulons l'hypothèse que l'accès à des sources variées d'informations (c'est-à-dire au dossier médical en plus de l'entretien) limitera les biais liés à l'évaluation. Il semble difficile de prédire plus en amont les résultats d'une telle recherche qui constitue à notre connaissance l'unique étude sur la question.

\section{MÉTHODE}

\section{Population}

L'échantillon est composé de 128 détenus majeurs, de sexe masculin, ayant commis des faits de violence. Les participants de cet échantillon ont en moyenne 44,2 ans (SD : 12,3). Leur niveau d'étude moyen correspondant au BEP (moyenne : 10,3 (SD : 2,5), valeurs minimale et maximale : 1-15). Ils ont été condamnés à une peine moyenne de 128 mois de détention (SD : 84,2) et ont effectué au moment du recueil de données en moyenne 58,9 mois de détention (SD : 53,1).

La population est répartie en deux groupes : un groupe d'Auteurs d'Infractions à Caractère Sexuel (auteurs d'infractions à caractère sexuel, $n=86,67,2 \%$ ) et un groupe d'auteurs de Violence Non Sexuelle (VNS, $n=41,32,8 \%$ ). Pour chaque groupe, deux conditions expérimentales ont été proposées : une condition «Entretien avec Dossier» $(E D)(n=43,33,6 \%)$ composée des participants pour lesquels la cotation des échelles de risque s'est faite à l'aide d'un entretien semi-structuré et de la consultation des dossiers médicaux. Les dossiers médicaux de ce groupe comprenaient en moyenne 2,53 documents (1-5). Et une condition «Entretien » $(\mathrm{E})(\mathrm{n}=85,66,4 \%)$ composée des participants pour lesquels la cotation des échelles de risque est limitée à un seul entretien semi-structuré. Le groupe «ED» présente significativement moins d'auteurs d'infractions à caractère sexuel $(\mathrm{n}=37$ pour le groupe $« \mathrm{ED} »$ et $\mathrm{n}=49$ pour le groupe $\ll \mathrm{E} », p<0,001)$.

\section{Instruments}

Afin de coter les échelles de risque deux sources d'informations ont été utilisées. Un entretien clinique semi-structuré, créé pour cette étude, abordait : (i) les antécédents judiciaires (dans l'enfance et à l'âge adulte), (ii) les faits en lien avec la condamnation actuelle (nombre et la nature de l'infraction, reconnaissance des faits...), (iii) les éléments psychopathologiques et somatiques (état mental actuel, antécédents de troubles...), 
(iv) les éléments sociaux et familiaux personnels (l'emploi, les relations amoureuses...), (v) les loisirs et activités (loisirs actuels, loisirs passés) et (vi) les projets d'avenir des participants (projets à venir, les

1 2 3 5 6 7 8

personnes ressources...). L'autorisation d'accès au dossier pénal étant impossible, seul le dossier médical a été utilisé comme source d'informations secondaires. Les dossiers médicaux contenaient tous au minimum des informations relatives à la prise en charge psychiatrique et/ou psychologique actuelle (fiche de traitement et attestations de suivi actuel).

Le Risk for Sexual Violence Protocol (RSVP) [26] est une échelle d'évaluation et de gestion du risque de récidive sexuelle. Elle évalue cinq domaines liés à la récidive. La version française a été traduite par Pham et Michaux (UMons). La cotation en trois possibilités est répétée pour le passé, le présent et l'avenir du participant et permet un score par domaine, ainsi qu'un score total. Elle présente de bonnes qualités psychométriques [27].

Le Historical, Clinical and Risk Management Scale-20 (HCR-20) [28] est une échelle d'évaluation et de gestion du risque de récidive violente. Elle évalue trois domaines liés à la récidive. La version française a été traduite et validée par Claix et Pham [29]. La cotation en trois possibilités permet le calcul d'un score dans chaque domaine, ainsi qu'un score total. Il présente de bonnes qualités psychométriques [30].

Le Level of Service/Case Management Inventory (LS/CMI) [31] permet d'évaluer le risque de récidive générale et la capacité de réinsertion des infracteurs. Il est composé de onze sections. La version française a été traduite et validée par Guay [32]. La section 1 (seule utilisée dans cette étude) évalue huit domaines liés à la récidive. Il est possible de scorer chaque domaine et un score total. L'échelle présente de bonnes qualités psychométriques [33].

La Social desirability Scale (SDS-17) [34], en auto-questionnaire, permet d'évaluer la tendance à la désirabilité sociale. La version française de la SDS-17 permet l'obtention d'un score total [35]. Cette échelle présente de bonnes qualités psychométriques [34].

Le Mini International Neuropsychiatric Interview (MINI) [36], en entretien semi-structuré, évalue la présence de troubles psychiatriques de l'axe 1 (troubles de l'humeur, anxieux, phobiques, obsessionnels, dépendance et abus de substances, troubles alimentaires, psychotiques) et du trouble de la personnalité 
antisociale (axe 2) selon les critères du DSM-IV [37]. La version française a été traduite par Lecrubier et al

[38].

1

2

\section{Procédure}

L'ensemble de la procédure est présenté en figure 1. Cette étude a reçu un avis favorable (n²017-077) du groupe éthique d'aide à la recherche clinique pour les protocoles de recherche non soumis au comité de protection des personnes (ERERC). Les passations ont été réalisées au sein de services sanitaires de quatre établissements pénitentiaires de régions françaises différentes entre mars 2018 et février 2020. L'ensemble des entretiens semi-structurés ont été réalisés par un évaluateur, psychologue clinicien, formé à l'utilisation des échelles de risque proposées dans cette étude. Les informations collectées lors des entretiens semistructurés ont permis la cotation des trois échelles de risque. La RSVP, qui permet l'appréciation d'un risque de récidive uniquement sexuel, n'a été cotée que pour les auteurs d'infractions à caractère sexuel. Une fois l'entretien semi-structuré proposé au participant, les dossiers médicaux pouvaient être consultés par le même expérimentateur. Les participants du groupe «E » avaient dans leur dossier les informations médicales générales dégagées à partir de l'ensemble des dossiers. Les participants du groupe «ED»avaient dans leur dossier des informations supplémentaires (expertises psychiatriques et psychologiques, comptes rendus d'hospitalisations en psychiatrie, ordonnances de condamnations définitives, fiches pénales). Le contexte des soins médicaux et psychiatriques dans des milieux pénitentiaires (donc judiciaires) peut amener les soignants à accéder à des informations judiciaires (comme notamment l'expertise psychiatrique) qui sont alors parfois contenues dans les dossiers médicaux des patients.

Afin de s'assurer de la bonne fidélité interjuge des scores au RSVP, HCR-20 et LS/CMI, une double cotation à l'aveugle a été réalisée pour dix participants $(7,81 \%)$, sélectionnés au hasard, par un second chercheur formé à la cotation des échelles de risque et exerçant dans un service médico-légal belge. La fidélité interjuge est satisfaisante pour les scores totaux aux échelles (score total de la RSVP : $\rho=0,96 ; p<0,01$; score total au HCR-20 : $\rho=0,93 ; p<0,01 ;$ score total au LS/CMI : $\rho=0,86 ; p<0,01)$.

\section{Analyses statistiques}

L'ensemble des analyses ont été réalisées à l'aide du logiciel IBM@ SPSS@ version 25. La distribution de notre population ne suit pas une loi normale, c'est pourquoi les analyses réalisées sont non paramétriques. Des comparaisons de moyennes non paramétriques (Test de Mann Whitney) ont été réalisées pour l'ensemble 
des résultats. Seules les comparaisons de présence de pathologies psychiatriques passées, actuelles et vie entière au sein des groupes « $\mathrm{E} »$ et « $\mathrm{ED} »$ des auteurs d'infractions à caractère sexuel et des auteurs de

1 2 3

violences non sexuelle ont été analysées à l'aide de $\mathrm{Chi}^{2}$. Les résultats sont présentés sous la forme : moyenne [écart-type] et par défaut la significativité est considérée à $p<0,05$. Nous avons fait le choix de scinder notre population selon le motif actuel de condamnation pour obtenir un groupe d'auteurs d'infractions à caractère sexuel et un groupe d'auteurs de violences non-sexuelle. Ainsi, le risque d'une population trop hétérogène est écarté.

\section{RÉSULTATS}

Comparaisons des scores totaux aux échelles de risque des groupes «ED » et «E » chez les auteurs d'infractions à caractère sexuel et les auteurs de violences non-sexuelles

Que ce soit pour les auteurs d'infractions à caractère sexuel comme les auteurs de violences non sexuelles, les groupes « $\mathrm{ED} »$ et « $\mathrm{E} »$ sont équivalents au niveau de l'âge, le niveau d'études, la durée de condamnation, la durée de détention effectuée, la désirabilité sociale évaluée à l'aide de la SDS-17 et la présence de troubles psychiatriques passés, actuels et vie entière, évalués à l'aide du MINI.

Le tableau 1 présente les résultats aux trois échelles RSVP, HCR-20 et RSVP chez les auteurs de violences non sexuelle et auteurs d'infractions à caractère sexuel. On observe des résultats significatifs seulement pour les échelles RSVP et LS/CMI et parmi le groupe des auteurs d'infractions à caractère sexuel. Aucune différence significative n'est observée pour la HCR-20 et chez les auteurs de violences non sexuelles. Les résultats suivants se concentreront uniquement sur les auteurs d'infractions à caractère sexuel et les échelles RSVP et LS/CMI.

Comparaisons des scores aux items de risque des groupes « $E D$ » et « $E$ » chez les auteurs d'infractions à caractère sexuel seuls

La suite des résultats se focalise sur les différences entre les items de la RSVP et du LS/CMI pour les groupes « $\mathrm{ED} \gg$ et «E $\gg$ chez les auteurs d'infractions à caractère sexuel. Seules les différences significatives sont rapportées Tableau 2. On observe que le groupe «ED» présente des scores significativement plus faibles aux items «Antécédents Violences Sexuelles», «Coercition physique-Récent (4R)», «Coercition psychique-Récent $(5 \mathrm{R}) »$, «Coercition psychique-Futur (5F) », «Ajustement social-Récent », « Ajustement 
social-Futur », «Emploi-Futur $(18 \mathrm{~F}) »$, « Gestion-Récent », « Gestion-Futur » de la RSVP et « Actuellement sans emploi (item 9)», «Pas emploi pendant 1 an (item 11)», «Participation au rendement (item 15)»,

1 2 3 4 5 6 7 8

«Rapports aux pairs (item 16) », « Rapports à l'autorité (item 17) », « Tendance à vivre des problèmes (item 43)» du LS/CMI. Le groupe «ED» présente des scores significativement plus élevés aux items «Antécédents VS-Antérieur », «Chronicité-Antérieur (1A)», «Déviance-Antérieur (11A)», «DévianceRécent $(11 \mathrm{R}) \gg$ et «Déviance-Futur $(11 \mathrm{~F}) »$ de la RSVP et « Nombre d'infractions actuelles (item 4) » du LS/CMI.

\section{DISCUSSION}

Les résultats aux échelles de risque montrent que $(i)$ les scores totaux ne diffèrent pas selon l'analyse des dossiers en plus d'un entretien semi-structuré et (ii) que lorsque des résultats sont significatifs, ils vont majoritairement dans le sens d'une évaluation plus faible du score de risque. Ce résultat est cohérent avec l'étude de Quesada et al. [39] qui a montré une diminution des scores de risque lorsque l'évaluation était réalisée par des praticiens ayant eu accès à des entretiens avec les évalués en plus de la lecture du dossier en comparaison aux chercheurs ayant côtés les échelles uniquement sur dossier. En France, l'utilisation d'échelles de risque, et en particulier des échelles qui permettant de catégoriser les infracteurs selon leur niveau de risque, entraînent chez les professionnels la crainte d'une potentielle stigmatisation des infracteurs 40]. Leur perception des outils est souvent réduite aux échelles actuarielles ne comprenant que des items passés et irréversibles [41]. Ces échelles stigmatiseraient les infracteurs car (i) elles les placeraient dans une catégorie type (à risque ou non) et (ii) leur caractère statique ne permettrait pas aux infracteurs d'évoluer et majorerait ainsi la perception du risque [40]. Notre étude s'appuie premièrement sur des échelles de jugement professionnel structuré et de jugement clinique intégré, basés sur des facteurs statiques mais également dynamiques, qui ont la capacité de considérer les évolutions positives de l'infracteur. De plus, les scores totaux de notre étude suggèrent que l'utilisation des dossiers médicaux n'amène pas à une évaluation plus élevée du risque de récidive. Ainsi, l'utilisation des dossiers médicaux en plus de l'entretien engendrerait une évaluation plus faible du risque de récidive. Ceci est cohérent avec (i) les travaux de la littérature soulignant que l'utilisation d'échelles structurées limite permet les faux positifs qui sont prévalant via le jugement clinique 
non structuré [4] et confirme ainsi (ii) les recommandations de bonnes pratiques des échelles préconisant l'utilisation de différentes sources d'informations pour une meilleure objectivité [42].

Deuxièmement, nous observons qu'à la suite de la consultation des dossiers, les scores de facteurs présents et futurs sont plus faibles. Sans doute la détention est un environnement particulier qui incite à développer certains mécanismes de défenses notamment chez les infracteurs ayant commis des faits graves (comme des violences sexuelles) [43]. La culture de la «méfiance»va entraîner une modification de la relation aux autres qui peut passer par le fait de se montrer plus «dangereux » [43]. Ceci a pu amener la personne évaluée à se montrer sous un jour plus négatif qu'il ne l'est en réalité, majorant certains facteurs comme la coercition physique et psychique utilisée lors des faits récents. Par ailleurs, des biais cognitifs peuvent impacter la compréhension de la situation par l'évaluateur. Le biais de saillance entraîne l'évaluateur à considérer que ce qui est saillant comme plus fréquent [42]. Les événements présents peuvent ainsi devenir plus saillants pour l'évaluateur (par exemple, les faits récents) car plus manifestes et nombreux dans la mémoire et le discours des personnes évalués. Les événements futurs peuvent être perçus comme plus saillants selon l'évaluateur car ils constituent sa préoccupation première dans l'appréciation du risque de violence.

Troisièmement, la consultation des dossiers semble augmenter les scores des facteurs passés. L'évaluateur peut avoir tendance, en étant tributaire des données auto rapportés, toujours à cause du biais cognitif de saillance, à sous-estimer le poids des éléments du passé [16]. De plus, la détention peut atténuer les fonctions cognitives des auteurs d'infractions à caractère sexuel et cet impact augmente avec la durée de détention [44]. Ainsi, des problèmes mnésiques chez les infracteurs pourraient engendrer des difficultés à relater des informations passées (comme les antécédents de violences sexuelles).

Enfin, à la différence d'items « factuels » (comme l'emploi ou les loisirs) la consultation des dossiers semble augmenter les scores de certains items «plus impliquant» car chargés émotionnellement comme le nombre d'infractions actuelles et la déviance (antérieure, récente, future). La déviance est définie par la présence d'intérêts, de préférences, d'activation ou de comportements sexuels qui impliquent une focalisation sur un objet ou une personne inappropriée. L'évaluation de ces informations pourrait être plus difficile car elle nécessite de poser des questions relatives à la sexualité déviante. La situation d'évaluation renforce la désirabilité sociale c'est-à-dire la tendance à se présenter soi-même d'une manière qui sera valorisée par autrui 45]. Les infracteurs vont alors être réticents à révéler certains faits qui pourraient soit contrecarrer cette 
recherche d'approbation soit générer du rejet chez l'autre [45]. D'autant que le présent échantillon est composé d'auteurs d'infractions à caractère sexuel, des infracteurs particulièrement sensibles aux regards d'autrui [46].

1 2 3 4 5 6 7 8

Enfin, les cognitions « chaudes » [47] comme la déviance, ne peuvent survenir que dans des contextes précis. L'examen de documents reflétant l'historique des délits sexuels ou l'utilisation d'instruments psychométriques, qui pourraient être mentionnés dans les dossiers des auteurs d'infractions à caractère sexuel, sont nécessaires afin d'évaluer ces cognitions chaudes [47,48].

Cette étude a tenté de montrer que la consultation de sources d'informations variées comme l'apport des dossiers médicaux contenant des informations médicales mais aussi parfois judiciaires rééquilibre le poids de plusieurs facteurs en limitant les effets subjectifs de la personne évaluée, de l'évaluateur, de la rencontre entre les deux et de la nature de certaines informations et en fournissant à l'évaluateur des données plus solides, car probablement convergentes pour la cotation des items. L'étude présente des limites. Notre échantillon est composé de auteurs de violences non-sexuelles et d'auteurs d'infractions à caractère sexuel. Aucune différence n'a été observé entre le groupe « $\mathrm{ED} »$ et le groupe « $\mathrm{E} »$ chez les auteurs de violences nonsexuelles. Des différences ont été observées entre le groupe « $\mathrm{ED} »$ et le groupe «E $»$ uniquement chez les auteurs d'infractions à caractère sexuel. L'échantillon considéré pour l'étude des résultats a, donc, été réduit aux auteurs d'infractions à caractère sexuel uniquement. Ceci peut être dû à la présence plus importante chez les auteurs d'infractions à caractère sexuel de troubles personnels [49] (impactant la rencontre avec l'autre et rendant l'évaluation sans donnée objective plus difficile). De plus, parmi diverses infractions, les faits de violences sexuelles génèrent souvent chez les interlocuteurs davantage de représentations négatives, qui induisent une perception spontanée plus négative des auteurs d'infractions à caractère sexuel [50]. Ceci concerne plus particulièrement les agresseurs sexuels d'enfants [51], qui composent une partie importante de notre population. Bien que ces représentations négatives soient moins présentes chez les soignants [51], elles impacteraient néanmoins l'évaluation des auteurs d'infractions à caractère sexuel et de leur risque. Des sources d'informations plus variées permettraient de nuancer et contrebalancer ces perceptions négatives, pour une évaluation plus objective. De plus, la taille de l'échantillon des auteurs d'infractions à caractère sexuel dans les groupes « Dossier» et «Entretien » demeure limitée. D’autres études identiques sur des échantillons plus importants faciliteraient la généralisation des résultats. Par ailleurs, la formation des groupes « $\mathrm{E} »$ et « $\mathrm{ED}$ » a été réalisée en fonction du contenu des dossiers médicaux des participants. À l'avenir, une assignation 
aléatoire pourrait définir ces groupes et limiterait ainsi les biais. Cette étude a été réalisée dans un contexte particulier qu'est la détention. Selon une approche développementale, le risque de récidive serait le fruit de

1

facteurs développementaux qui induiraient des facteurs de risque psychologiques de vulnérabilité qui a leur tour dans des contextes spécifiques entraîneraient la récidive [52]. Or la prison est un environnement qui limite l'exposition à certains facteurs contextuels de risque, comme la présence de potentielles victimes mineures. De plus, l'absence de données de récidive est une limite supplémentaire à l'étude. Elles permettraient de vérifier si l'utilisation de sources d'informations variées améliorent ou non la prédiction des échelles de risque. Mais leur recueil n'a pas été possible dans la mesure où l'évaluation de la récidive est possible sur une période d'observation longue après la sortie de détention (par exemple, deux ans pour l'étude de [5]). Au moment de la collecte des données, qui s'est achevée en 2020, notre échantillon, qui a été condamné à de lourdes peines, avait purgé un peu plus de la moitié des peines $(45,60 \%)$. Les participants seront libérés de détention en moyenne 69,66 mois après notre évaluation. Il n'a donc pas été possible d'évaluer nos participants au moment de leur libération afin de déterminer s'ils avaient effectivement été condamnés à nouveau. La principale limite est la composition des dossiers médicaux. En effet, nous avons pu constater une hétérogénéité importante du contenu des dossiers en matière de (i) quantité d'informations (certains dossiers ne contiennent que des informations actuelles tandis que d'autres retracent le passé de l'infracteur) et (ii) qualité des informations (certains dossiers ne contiennent uniquement que les éléments médicaux tandis que d'autres contiennent des documents pénaux). Les informations sociales sont souvent inexistantes. Ce constat avait déjà été partagé dans une étude montrant (i) le caractère hétérogène des contenus de 69 expertises françaises de pédopornographes et (ii) l'absence de certaines données notamment en matière d'évaluation du risque de récidive [53]. Pourtant certaines informations sont indispensables pour la cotation des échelles de risque : les évaluations passées, les rapports psychologiques et psychiatriques ou encore le casier judiciaire [42]. Dans d'autres pays, comme au Canada, il est même possible de s'entretenir avec des proches de la personne évaluée (comme des membres de la famille, des professionnels... [16] ce qui ne constitue pas une pratique courante en France.

\section{CONCLUSION}

Cette étude a tenté de mesurer l'impact de l'utilisation, sur les scores aux échelles de risque, des informations contenus ou non dans les dossiers médicaux de 128 détenus violents. Les résultats ont montré 
que l'utilisation des informations contenues dans les dossiers médicaux associée à un entretien entraînait ( $i)$

des variations de scores de risque uniquement pour les auteurs d'infractions à caractère sexuel et uniquement

1 2 3

pour les échelles RSVP et LS/CMI, (ii) des scores totaux plus faibles de risque de récidive, (ii) des scores plus faibles à certains facteurs de risque présents et futurs, (iii) des scores plus élevés à certains facteurs de risque passés et enfin, (iv) des scores plus élevés à certains facteurs « plus impliquant » et chargés émotionnellement.

En France, l'utilisation des échelles de risque génère une grande réticence de la part des professionnels mais son implantation est en cours. Une utilisation optimale de ces échelles n'est rendue possible qu'avec l'accès aux données qui permettent de coter l'ensemble des facteurs de risque composant les échelles. Mais dans le contexte français, le secret professionnel (par ailleurs indispensable à la pratique), l'hétérogénéité qualitative et quantitative complique l'utilisation des échelles. Les résultats montrent que l'utilisation de certaines données impacte les scores des échelles destinées à apprécier le risque de récidive ainsi que l'instauration de la prise en charge.

\section{Les auteurs déclarent ne pas avoir de liens d’intérêts.}

\section{BIBLIOGRAPHIE}

[1] Elbogen EB, Huss MT. The role of serotonin in violence and schizophrenia: Implications for risk assessment. J Psychiatry Law 2000;28:19-48.

[2] Neal T, Grisso T. Assessment Practices and Expert Judgment Methods in Forensic Psychology and Psychiatry: An International Snapshot. Crim Justice Behav 2014;41:1406-21. https://doi.org/10.1177/00093854814548449.

[3] Quinsey VL. The prediction and explanation of criminal violence. Int J Law Psychiatry 1995;18:11727. https://doi.org/10.1016/0160-2527(95)00001-X.

[4] Melton GB, Petrila J, Poythress N, Slobogin C. Psychological evaluations for the courts : a handbook for mental health professionnals and lawyers. New York, State of NY, US: Guilford Press; 2007.

[5] Wormith JS, Hogg S, Guzzo L. The Predictive Validity of a General Risk/Needs Assessment Inventory on Sexual Offender Recidivism and an Exploration of the Professional Override. Crim Justice Behav 2012;39:1511-38. https://doi.org/10.1177/0093854812455741.

[6] Webster CD, Douglas KS, Eaves D, Hart SD. HCR-20: Assessing risk for violence, version 2. Burnaby, Canada: Simon Fraser University; 1997.

[7] Pham T-H, Ducro C, Desmarais SL, Hurducas C, Arbach-Lucioni K, Condemarin C, et al. Enquête internationale sur les pratiques d'évaluation du risque de violence : présentation des données belges. Ann Méd-Psychol 2016;7:539-43.

[8] Singh JP, Grann M, Fazel S. A comparative study of violence risk assessment tools: A systematic review and metaregression analysis of 68 studies involving 25,980 participants. Clin Psychol Rev 
2011;31:499-513. https://doi.org/10.1016/j.cpr.2010.11.009.

[9] Singh JP, Desmarais SL, Hurducas C, Arbach-Lucioni K, Condemarin C, Dean K, et al. International Perspectives on the Practical Application of Violence Risk Assessment: A Global Survey of 44 Countries. Int J Forensic Ment Health 2014;13:193-206.

https://doi.org/10.1080/14999013.2014.922141.

10] Hanson RK, Thornton D. Improving risk assessment for sex offenders : a comparison of three actuarial scales. Law Hum Behav 2000;24:119-36.

[11] Hogan NR, Olver ME. Assessing risk for aggression in forensic psychiatric inpatients: An examination of five measures. Law Hum Behav 2016;40:233-43. https://doi.org/10.1037//hb0000179.

[12] Hanson RK, Morton-Bourgon KE. The accuracy of recidivism risk assessments for sexual offenders: A meta-analysis of 118 prediction studies. Psychol Assess 2009;21:1-21. https://doi.org/10.1037/a0014421.

[13] Flores AW, Lowenkamp CT, Holsinger AM, Latessa EJ. Predicting outcome with the Level of Service Inventory-Revised: The importance of implementation integrity. J Crim Justice 2006;34:523-9. https://doi.org/10.1016/j.jcrimjus.2006.09.007.

[14] Hart SD. Assessing risk for sexual violence: The Risk for Sexual Violence Protocol (RSVP) 2003.

[15] Hanson RK. How should risk assessments for sexual offenders be conducted? 2007.

[16] Guay J-P. Évaluer le risque de récidive: un état de la recherche et des principaux enjeux pratiques. Paris, France: Conférence de consensus; 2012.

[17] Moulin, V, Gholamrezaee MM, Grassier J, Abbiati M. Le processus d'évaluation du risque de récidive en consensus : intérêts et limites. Ann Méd-Psychol 2020;178:540-7.

[18] Drevon E, Maurel D, Dufour C. Veille stratégique et prise de décision : une revue de la littérature. Doc Bibl 2018;64:28-34. https://doi.org/10.7202/1043720ar.

[19] West O'Donell N. Decision-Making Ability Beliefs: Determinants of Pre-experiential Choice Confidence and Resistance to Choice Change. The Ohio State university, 2018.

[20] Douglas KS, Ogloff JRP. The Impact of Confidence on the Accuracy of Structured Professional and Actuarial Violence Risk Judgments in a Sample of Forensic Psychiatric Patients. Law Hum Behav 2003;27:573-87. https://doi.org/10.1023/B:LAHU.0000004887.50905.f7.

[21] Herpin N. Le dossier pénal et son double. Sociol Trav 1981;23:44-9. https://doi.org/10.3406/sotra.1981.1661.

[22] Décret n $99-276$ relatif à la création des services pénitentiaires d'insertion et de probation. 1999.

[23] Décret $n^{\circ}$ 2002-637 relatif à l'accès aux informations personnelles détenues par les professionnels et les établissements de santé. 2002.

[24] Article R1112-2. vol. R1112-2. 2016.

[25] Montet I. Le dossier médical en psychiatrie ambulatoire. Inf Psychiatr 2008;84:354-60.

[26] Hart SD, Kropp PR, Laws DR, Klaver J, Logan C, Watt KA. The risk for sexual violence protocol (RSVP): structured professional guidelines for assessing risk of sexual violence. Burnaby, Canada: Mental Health, Law, and Policy Institute, Simon Fraser University; 2003.

[27] Hart SD, Boer DP. Structured professional judgment guidelines for sexual violence risk assessment: The Sexual Violence Risk-20 (SVR-20) and Risk for Sexual Violence Protocol (RSVP). Handb. Violence Risk Assess., New York, State of NY, US: Routledge, Taylor \& Francis Group; 2010, p. 269-94.

[28] Webster CD, Eaves D, Douglas KS, Wintrup A. The HCR-20 Scheme: The Assessment of Dangerousness and Risk (Version 1). Burnaby, B.C., Canada: Simon Fraser University and Forensic Psychiatric Services Commission of British Columbia; 1995.

[29] Claix A, Pham T-H. Evaluation of the HCR-20 Violence Risk Assessment Scheme in a Belgian 
forensic population. L'Encephale 2004;30:447-53. https://doi.org/10.1016/s0013-7006(04)95459-0.

[30] Douglas KS, Hart SD, Webster CD, Belfrage H, Guy LS, Wilson CM. Historical-Clinical-Risk Management-20, Version 3 (HCR-20V3): Development and overview. Int J Forensic Ment Health 2014;13:93-108. https://doi.org/10.1080/14999013.2014.906519.

[31] Andrews DA, Bonta JL, Wormith JS. The Level of Service/Case Management Inventory (LS/CMI). Crime Delinquency 2004:7-27.

[32] Guay J-P. L'évaluation du risque et des besoins criminogènes à la lumière des données probantes: Une étude de validation de la version Française de l'inventaire de niveau de service et de gestion des casLS/CMI. Eur Rev Appl Psychol 2016;66:199-210. https://doi.org/10.1016/j.erap.2016.04.003.

[33] Wormith JS, Hogg SM, Guzzo L. The predictive validity of the LS/CMI with aboriginal offenders in Canada. Crim Justice Behav 2015;42:481-508. https://doi.org/10.1177/0093854814552843.

[34] Stöber J. The Social Desirability Scale-17 (SDS-17). Eur J Psychol Assess 2001;17:222-32. https://doi.org/10.1027//1015-5759.17.3.222.

[35] Blais MR, Lachance L, Riddle A. Validation de la version française de la mesure de la désirabilité sociale de Marlowe-Crowne. Université de Montréal, Montréal, Canada; 1991.

[36] Sheehan DV, Lecrubier Y, Sheehan KH, Amorim P, Janavs J, Weiller E, et al. The Mini-International Neuropsychiatric Interview (M.I.N.I.): the development and validation of a structured diagnostic psychiatric interview for DSM-IV and ICD-10. J Clin Psychiatry 1998;59 Suppl 20:22-33;quiz 34-57.

[37] American Psychiatric Association. Diagnostic and Statistical Manual of Mental Disorders, 4th Edition. (DSM IV). American Psychiatric Association. Washington DC.; 1994.

[38] Lecrubier Y, Sheehan D, Weiller E, Amorim P, Bonora I, Sheehan K, et al. The Mini International Neuropsychiatric Interview (M.I.N.I.), a short diagnostic interview : Reliability and validity according to the CIDI. Eur Psychiatry 12 1997:232-41.

[39] Quesada S, Calkins C, Jeglic E. An Examination of the Interrater Reliability Between Practitioners and Researchers on the Static-99. Int J Offender Ther Comp Criminol 2014;58:1364-75. https://doi.org/10.1177/0306624X13495504.

[40] Moulin M, Palaric R, Gravier B. Quelle position professionnelle adopter face à la diversité des problèmes posés par l'évaluation des dangerosités ? Inf Psychiatr 2012:617-29. https://doi.org/10.3917/inpsy.8808.0617.

[41] Gravier, B, Moulin, V, Senon, JL. L'évaluation actuarielle de la dangerosité : impasses éthiques et dérives sociétales. Inf Psychiatr 2012:599-604. https://doi.org/10.3917/inpsy.8808.0599.

[42] Guay J-P, Benbouriche M, Parent G. L'évaluation structurée du risque de récidive des personnes placées sous-main de justice: Méthodes et enjeux. Prat Psychol 2015;21:235-57. https://doi.org/10.1016/j.prps.2015.05.005.

[43] Van Marle H. Mental Health Care in Prison: How to manage our care? Int J Prison Health 2005;3:11523.

[44] Combalbert N, Pennequin V. L'effet de l'âge et de la durée d'incarcération sur la santé mentale des détenus âgés. Ann Méd-Psychol Rev Psychiatr 2020;178:264-70. https://doi.org/10.1016/j.amp.2018.06.013.

[45] Aebi MF, Jaquier V. Les sondages de délinquance autoreportée : origines, fiabilité et validité. Deviance Soc 2008;32:205-27. https://doi.org/DOI : 10.3917/ds.322.0205.

[46] Tierney DW, McCabe MP. An evaluation of self-report measures of cognitive distortions and empathy among Australian sex offenders. Arch Sex Behav 2001;30:495-519. https://doi.org/10.1023/A:1010239217517.

[47] Benbouriche M, Vanderstukken O, Guay J-P, Testé B, Renaud P. Quelle pertinence pour l'évaluation standardisée des distorsions cognitives en délinquance sexuelle? Présentation, Illustrations, Limites, et Perspectives. Rev Int Criminol Police Tech Sci 2013;1:29-46. 
[48] Cortoni F, Longpré N. 9. Quelle est l'efficacité des traitements des agresseurs sexuels pour prévenir la récidive ? In: Lafortune D, Poupart J, Tanner S, editors. Quest. Criminol. Presses de 1'Université de Montréal, Montréal, Canada: 2018, p. 107-15.

1 [49] Chantry K, Craig RJ. Psychological screening of sexually violent offenders with the MCMI. J Clin Psychol 1994;50:430-5. https://doi.org/10.1002/1097-4679(199405)50:3<430::AIDJCLP2270500314>3.0.CO;2-W.

[50] Soldati L, Eytan A. Traitement des agresseurs sexuels : possibilités et limites. Rev Médicale Suisse 2014;10:647-50.

[51] Ferguson K, Ireland C. Attitudes towards sex offenders and the influence of offence type: A comparison of staff working in a forensic setting and students. Br J Forensic Pract 2006;8:10-9. https://doi.org/10.1108/14636646200600009.

[52] Beech AR, Ward T. The integration of etiology and risk in sexual offenders: A theoretical framework. Aggress Violent Behav 2004;10:31-63. https://doi.org/10.1016/j.avb.2003.08.002.

[53] Prat S, Jonas C. Analyse qualitative de rapports d'expertise psychiatrique concernant des faits de pornographie infantile. Ann Méd-Psychol 2015;173:148-52. 


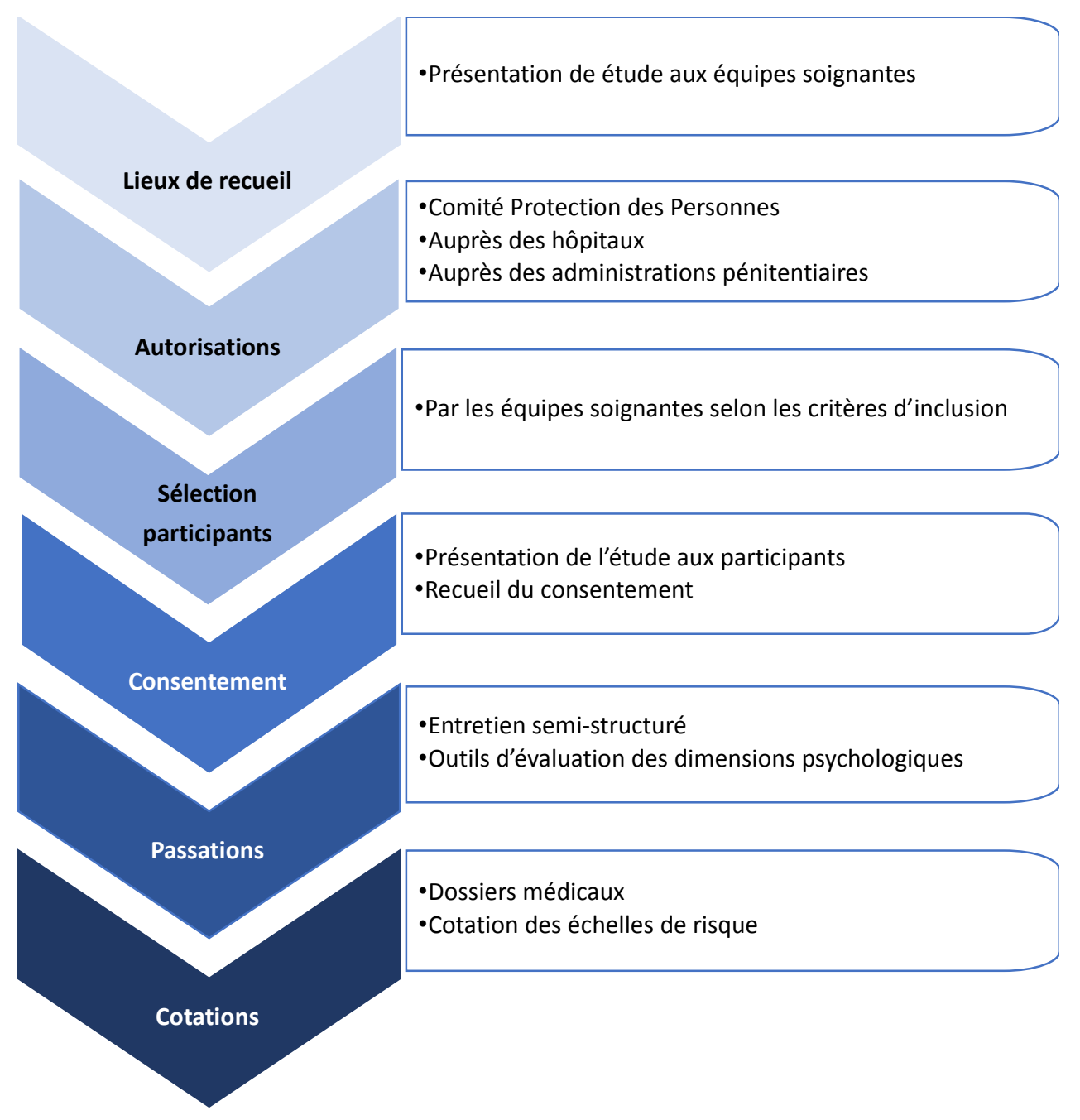


Tableau 1 : comparaisons des scores totaux aux échelles de risque entre les groupes « Entretien avec dossier » et « Entretien » chez les AICS et les VNS

\begin{tabular}{|c|c|c|c|c|c|c|}
\hline & \multicolumn{3}{|c|}{ AICS } & \multicolumn{3}{|c|}{ VNS } \\
\hline & $\begin{array}{c}\text { Entretien avec } \\
\text { Dossier }(\mathbf{n}=37) \\
\text { Moy. }[\mathrm{ET}]\end{array}$ & $\begin{array}{c}\text { Entretien } \\
(\mathbf{n}=49) \\
\text { Moy. [ET] }\end{array}$ & Test $\mathbf{U}$ & $\begin{array}{c}\text { Entretien avec } \\
\text { Dossier }(\mathbf{n}=36) \\
\text { Moy. }[\mathrm{ET}]\end{array}$ & $\begin{array}{c}\text { Entretien } \\
(\mathbf{n}=\mathbf{6}) \\
\text { Moy. [ET] }\end{array}$ & Test U \\
\hline Age & $47,43[10,41]$ & $44,43[11,36]$ & ns & $39,78[14,61]$ & $48,17[8,47]$ & ns \\
\hline Niveau étude & $10,51[2,57]$ & $9,82[2,65]$ & ns & $10,03[2,31]$ & $8,83[2,64]$ & ns \\
\hline Durée condamnation & $137,35[79,86]$ & $127,96[59,75]$ & ns & $\begin{array}{c}108,14 \\
{[107,55]}\end{array}$ & $\begin{array}{c}191,00 \\
{[107,76]}\end{array}$ & ns \\
\hline Durée peine effectuée & $75,73[70,28]$ & $49,81[31,32]$ & ns & $46,00[46,07]$ & $94,33[76,84]$ & ns \\
\hline Nombre de délits dans condamnation actuelle & $1,49[0,65]$ & $1,51[0,65]$ & ns & $1,28[0,74]$ & $1,17[0,41]$ & ns \\
\hline Désirabilité sociale & $10,87[2,80]$ & $9,75[3,10]$ & ns & $10,76[3,42]$ & $11,75[1,26]$ & ns \\
\hline RSVP- TOTAL & $46,4[15,5]$ & $50,2[15,9]$ & ns & - & - & - \\
\hline Antérieur Total & $20,4[6,2]$ & $18,2[6,46]$ & ns & - & - & - \\
\hline Récent Total & $11,9[5,2]$ & $16,2[6,3]$ & $0,02 *$ & - & - & - \\
\hline Futur Total & $14,1[5,3]$ & $15,8[5,1]$ & ns & - & - & - \\
\hline HCR- TOTAL & $12,7[6,8]$ & $14,0[6,6]$ & ns & $14,8[6,1]$ & $16,0[6,9]$ & ns \\
\hline Historique & $6,1[4,3]$ & $6,9[4,4]$ & ns & $7,0[3,6]$ & $8,6[4,9]$ & ns \\
\hline Clinique & $2,8[1,9]$ & $3,0[2,1]$ & ns & $4,0[1,7]$ & $3,6[2,0]$ & ns \\
\hline Risque & $3,7[1,9]$ & $4[2,1]$ & ns & $3,83[2,1]$ & $3,8[1,6]$ & ns \\
\hline LS/CMI- TOTAL & $14,2[6,4]$ & $16,7[6,3]$ & ns & $16,7[6,1]$ & $19,4[8,5]$ & ns \\
\hline Antécédents criminels & $3,6[1,8]$ & $3,4[2,0]$ & ns & $3,8[1,5]$ & $4,3[2,2]$ & ns \\
\hline Éducation \& emploi & $2,0[1,9]$ & $3,8[2,4]$ & $0,01 * *$ & $3,3[1,9]$ & $4,4[2,8]$ & ns \\
\hline Famille \& couple & $2,2[2,1]$ & $2,3[1,2]$ & ns & $1,5[0,8]$ & $2,0[1,2]$ & ns \\
\hline Loisirs \& activités récréatives & $2,7[0,8]$ & $2,8[0,8]$ & ns & $3,2[0,4]$ & $3,0[0,7]$ & ns \\
\hline Fréquentations & $0,6[0,7]$ & $0,9[0,8]$ & ns & $0,7[0,8]$ & $0,9[0,7]$ & ns \\
\hline Alcool \& drogues & $1,3[1,9]$ & $1,1[1,5]$ & ns & $1,8[1,7]$ & $2,0[1,9]$ & ns \\
\hline Attitudes. \& orientation criminelles & $1,1[1,0]$ & $1,2[0,9]$ & ns & $1,0[0,9]$ & $1,4[1,2]$ & ns \\
\hline Comportement antisocial & $0,7[1,0]$ & $1,2[1,0]$ & $0,01 * *$ & $1,3[1,8]$ & $1,4[1,2]$ & ns \\
\hline
\end{tabular}

ns : non significatif ; ${ }^{*} p<0,05 ; * * p<0,01$

RSVP : Risk for Sexual Violence Protocol ; HCR : Historical, Clinical and Risk Management Scale - 20 ; LS/CMI : Level of Service/Case Management Inventory 
Tableau 2 : détails des comparaisons des items de risque de la RSVP et du LS/CMI entre les groupes « Entretien avec dossier » et « Entretien » chez les AICS

\begin{tabular}{|c|c|c|c|}
\hline & $\begin{array}{c}\begin{array}{c}\text { Entretien avec Dossier } \\
(\mathbf{n}=\mathbf{3 7}) \\
\text { Moy. [ET] }\end{array} \\
\end{array}$ & $\begin{array}{c}\begin{array}{c}\text { Entretien } \\
(\mathbf{n}=49) \\
\text { Moy. [ET] }\end{array} \\
\end{array}$ & Test $\mathbf{U}$ \\
\hline \multicolumn{4}{|l|}{ RSVP } \\
\hline Antécédents VS - Antérieur & $3,6[2,5]$ & $1,8[2,2]$ & $0,01 * *$ \\
\hline Antécédents VS - Récent & $0,4[0,9]$ & $2,0[1,6]$ & $0,01 * *$ \\
\hline Chronicité Antérieur (1A) & $1,1[0,9]$ & $0,6[0,8]$ & $0,01 * *$ \\
\hline Escalade Antérieur (3A) & $0,5[0,8]$ & $0,1[0,5]$ & $0,01 * *$ \\
\hline Coercition physique Récent (4R) & $0,1[0,4]$ & $0,6[0,9]$ & $0,01 * *$ \\
\hline Coercition psychologique Récent (5R) & $0,2[0,6]$ & $1,2[1,0]$ & $0,01 * *$ \\
\hline Coercition psychologique Futur (5F) & $0,5[0,6]$ & $0,8[0,7]$ & $0,04 *$ \\
\hline Déviance Antérieur (11A) & $0,9[0,8]$ & $0,5[0,8]$ & $0,01 * *$ \\
\hline Déviance Récent (11R) & $0,8[0,8]$ & $0,4[0,7]$ & $0,01 * *$ \\
\hline Déviance Futur (11F) & $0,8[0,8]$ & $0,4[0,7]$ & $0,01 * *$ \\
\hline Ajustement social - Récent & $3,2[1,9]$ & $4,2[2,1]$ & $0,02 *$ \\
\hline Ajustement social - Futur & $3,3[1,8]$ & $4,2[1,9]$ & $0,03 *$ \\
\hline Emploi Futur (18F) & $0,6[0,8]$ & $1,0[0,8]$ & $0,04 *$ \\
\hline Gestion - Récent & $1,2[1,3]$ & $2,1[1,9]$ & $0,02 *$ \\
\hline Gestion - Futur & $1,4[1,2]$ & $2,3[1,7]$ & $0,03 *$ \\
\hline Planification Récent (20R) & $0,5[0,8]$ & $1,1[0,9]$ & $0,01 * *$ \\
\hline Planification Futur (20F) & $0,5[0,7]$ & $1,2[0,8]$ & $0,01 * *$ \\
\hline \multicolumn{4}{|l|}{ LS/CMI } \\
\hline Nombre d'infractions actuelles (Item 4) & $0,5[0,5]$ & $0,2[0,4]$ & $0,02 *$ \\
\hline Actuellement sans emploi (Item 9) & $0,2[0,4]$ & $0,5[0,5]$ & $0,03 *$ \\
\hline Pas emploi pendant 1an (Item 11) & $0,1[0,2]$ & $0,20,4]$ & $0,02 *$ \\
\hline Participation ou rendement (Item 15) & $0,2[0,4]$ & $0,5[0,5]$ & $0,01 * *$ \\
\hline Rapports aux pairs (Item 16) & $0,2[0,4]$ & $0,5[0,5]$ & $0,01 * *$ \\
\hline Rapports à l'autorité (Item 17) & $0,1[0,5]$ & $0,5[0,5]$ & $0,01 * *$ \\
\hline Tendance à vivre des problèmes (Item 43) & $0,3[0,5]$ & $0,6[0,5]$ & $0,01 * *$ \\
\hline
\end{tabular}

ns : non significatif ; ${ }^{*} p<0,05 ; * * p<0,01$

RSVP : Risk for Sexual Violence Protocol ; HCR : Historical, Clinical and Risk Management Scale - 20 ; LS/CMI : Level of Service/Case Management Inventory 